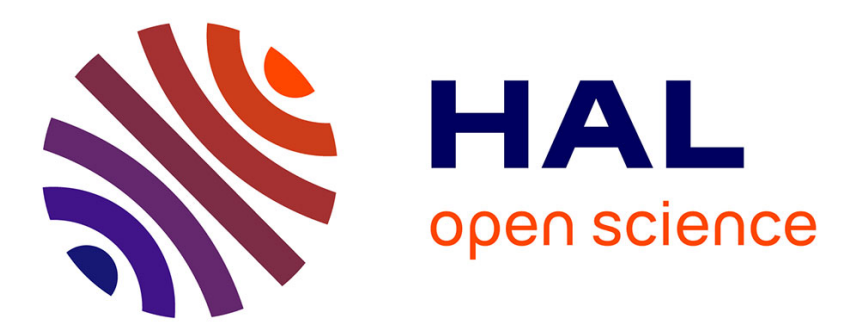

\title{
Estimation of temporal parameters during sprint running using a trunk-mounted inertial measurement unit
}

Elena Bergamini, Pietro Picerno, Helene Pillet, Françoise Natta, Patricia Thoreux, Valentina Camomilla

\section{To cite this version:}

Elena Bergamini, Pietro Picerno, Helene Pillet, Françoise Natta, Patricia Thoreux, et al.. Estimation of temporal parameters during sprint running using a trunk-mounted inertial measurement unit. Journal of Biomechanics, 2012, 45, pp.1123-1126. 10.1016/j.jbiomech.2011.12.020 . hal-01083834

\section{HAL Id: hal-01083834 https://hal.science/hal-01083834}

Submitted on 18 Nov 2014

HAL is a multi-disciplinary open access archive for the deposit and dissemination of scientific research documents, whether they are published or not. The documents may come from teaching and research institutions in France or abroad, or from public or private research centers.
L'archive ouverte pluridisciplinaire HAL, est destinée au dépôt et à la diffusion de documents scientifiques de niveau recherche, publiés ou non, émanant des établissements d'enseignement et de recherche français ou étrangers, des laboratoires publics ou privés. 


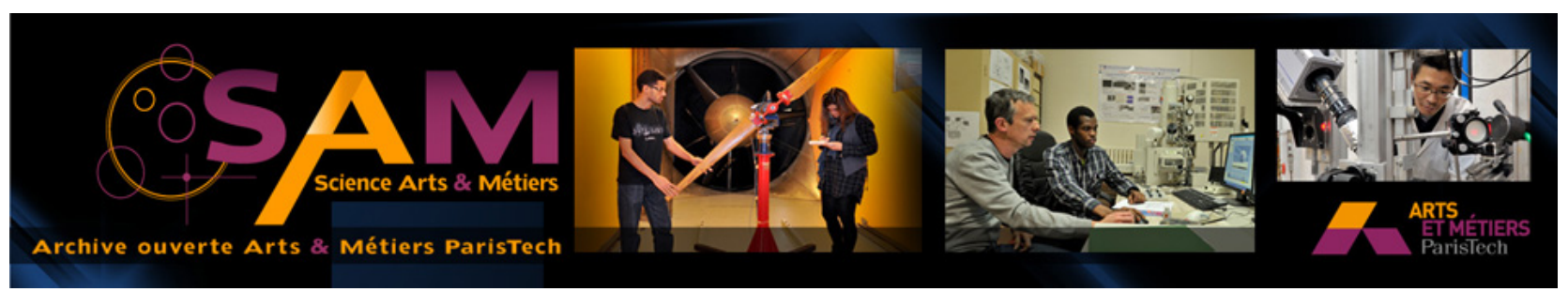

\section{Science Arts \& Métiers (SAM)}

is an open access repository that collects the work of Arts et Métiers ParisTech researchers and makes it freely available over the web where possible.

This is an author-deposited version published in: http://sam.ensam.eu

Handle ID: .http://hdl.handle.net/10985/8924

\section{To cite this version :}

Elena BERGAMINI, Pietro PICERNO, Helene PILLET, Francoise NATTA, Patricia THOREUX, Valentina CAMOMILLA - Estimation of temporal parameters during sprint running using a trunkmounted inertial measurement unit - Joural of Biomechanics - Vol. 45, p.1123-1126 - 2012 


\title{
Estimation of temporal parameters during sprint running using a trunk-mounted inertial measurement unit
}

\author{
Elena Bergamini ${ }^{\mathrm{a}, \mathrm{b}}$, Pietro Picerno ${ }^{\mathrm{a}}$, Hélène Pillet ${ }^{\mathrm{b}}$, Françoise Natta ${ }^{\mathrm{c}}$, Patricia Thoreux ${ }^{\mathrm{b}, \mathrm{d}}$, \\ Valentina Camomilla ${ }^{\text {a,* }}$ \\ a Locomotor Apparatus Bioengineering Laboratory, Department of Human Movement and Sport Sciences, University of Rome “Foro Italico“, piazza Lauro de Bosis 15, 00135 \\ Rome, Italy \\ ${ }^{\mathrm{b}}$ Laboratoire de Biomécanique, Arts et Métiers ParisTech, 151 bd de l'Hôpital, 75013 Paris, France \\ ' Service Recherche, Institut National du Sport, de l'Expertise et de la Performance, 11 av du Tremblay, 75012 Paris, France \\ ' Service de Chirurgie Orthopédique et Traumatologique, Hôpital Avicenne-Université Paris 13, 125 route de Stalingrad, 93009 Bobigny, France
}

Keywords:

Feature identification

Foot contact detection

Inertial sensor

Sports

Stance and stride duration

\begin{abstract}
A B S T R A C T
The purpose of this study was to identify consistent features in the signals supplied by a single inertial measurement unit (IMU), or thereof derived, for the identification of foot-strike and foot-off instants of time and for the estimation of stance and stride duration during the maintenance phase of sprint running. Maximal sprint runs were performed on tartan tracks by five amateur and six elite athletes, and durations derived from the IMU data were validated using force platforms and a high-speed video camera, respectively, for the two groups. The IMU was positioned on the lower back trunk (L1 level) of each athlete. The magnitudes of the acceleration and angular velocity vectors measured by the IMU, as well as their wavelet-mediated first and second derivatives were computed, and features related to foot-strike and foot-off events sought. No consistent features were found on the acceleration signal or on its first and second derivatives. Conversely, the foot-strike and foot-off events could be identified from features exhibited by the second derivative of the angular velocity magnitude. An average absolute difference of $0.005 \mathrm{~s}$ was found between IMU and reference estimates, for both stance and stride duration and for both amateur and elite athletes. The 95\% limits of agreement of this difference were less than $0.025 \mathrm{~s}$. The results proved that a single, trunk-mounted IMU is suitable to estimate stance and stride duration during sprint running, providing the opportunity to collect information in the field, without constraining or limiting athletes' and coaches' activities.
\end{abstract}

\section{Introduction}

Temporal parameters, i.e., flight and stance duration, during sprint running represent basic, yet very useful information for track and field coaches and their correlation with performance has been widely investigated in the literature (Čoh et al., 2006; Hunter et al., 2004a, 2004b). Previously, force platforms (Hunter et al., 2004b, 2005), stereophotogrammetric systems (Ciacci et al., 2010), optical bars (Čoh et al., 2006), or video-analysis (Ito et al., 2006) have been used to measure or estimate these parameters during sprint running. These instruments, however, are either limited in terms of acquisition volume or require long post-processing time.

As an alternative, wearable inertial measurement units (IMUs) allow in-the-field performance monitoring without constraining athletes' movements. Recently, stance duration has been estimated

\footnotetext{
*Corresponding author. Tel.: +3906 36733522; fax: +390636733517.

E-mail address: valentina.camomilla@uniroma4.it (V. Camomilla).
}

during sprint running using shank-mounted accelerometers (Purcell et al., 2005). However, more information concerning the overall strategy of the locomotor act could be provided mounting the unit close to the body centre of mass (Cavagna et al., 1971). Trunk-mounted IMUs have been used to estimate temporal parameters only during walking (Kavanagh and Menz, 2008) and distance running (Auvinet et al., 2002; Lee et al., 2010; Wixted et al., 2010), by identifying event-related features in the signals. During walking, for example, the peak forward acceleration of the centre of mass is related to foot-strike (Verkerke et al., 2005) or, during both walking and distance running, a repeatable change in the direction of trunk rotation about its longitudinal axis occurs prior to foot-off (Saunders et al., 2005; Schache et al., 2002).

However, robustness and reliability of any feature-identification approach rely on the accurate and consistent detection of such features within and across subjects. This highly depends on the signal-to-noise ratio, which, in turn, is influenced by the movements of the soft tissues separating the IMU from the skeleton. These movements are both subject-dependent 
Table 1

Gender $(\mathrm{F}=$ female, $\mathrm{M}=$ male), mass, and height of the six amateur $(\mathrm{A})$ and the five elite (E) athletes involved in the study. Average speed in the tested steps and, for elite sprinters, the personal best time on a $100 \mathrm{~m}$ run are also indicated.

\begin{tabular}{|c|c|c|c|c|c|c|c|c|c|c|c|}
\hline \multirow[b]{3}{*}{ Gender } & \multicolumn{6}{|c|}{ Group A } & \multicolumn{5}{|c|}{ Group E } \\
\hline & A1 & A2 & A3 & A4 & A5 & A6 & E1 & E2 & E3 & E4 & E5 \\
\hline & $\mathrm{F}$ & $\mathrm{F}$ & M & M & M & M & $\mathrm{F}$ & $\mathrm{F}$ & M & M & M \\
\hline Mass (kg) & 56 & 45 & 72 & 60 & 73 & 75 & 56 & 54 & 70 & 73 & 72 \\
\hline Height (m) & 1.71 & 1.48 & 1.72 & 1.83 & 1.78 & 1.80 & 1.70 & 1.74 & 1.74 & 1.80 & 1.86 \\
\hline Speed $(\mathrm{m} / \mathrm{s})$ & 5.7 & 7.0 & 7.0 & 7.4 & 6.4 & 7.4 & 9.7 & 9.7 & 10.8 & 9.9 & 9.8 \\
\hline Personal best (s) & $\#$ & $\#$ & $\#$ & $\#$ & $\#$ & $\#$ & 11.51 & 11.52 & 10.17 & 10.63 & 10.49 \\
\hline
\end{tabular}

(Lafortune et al., 1995) and sensitive to the site and method of the unit attachment (Forner-Cordero et al., 2008). Moreover, they can be assumed to be larger during sprint running due to the explosiveness of the task, thus jeopardising the consistency of the identified features.

The purpose of this study was to identify recognisable and consistent features in the waveform of the signals supplied by a lower trunk mounted IMU for the estimation of the stance and stride duration during the maintenance phase of sprint running. To this aim, maximal sprint runs were performed on tracks by both amateur and elite athletes. Data provided by an IMU were compared with force platform data or high-speed videos.

\section{Materials and methods}

Two groups of athletes participated in the study during two different experimental sessions, after giving their written informed consent, approved by the University Ethical Committee (Table 1).

The first experimental session involved six amateur athletes (group A). Tests were carried out on an indoor track. The facility was provided with six adjacent force platforms (Z20740AA, Kistler, Switzerland; total surface: $6.6 \times 0.6 \mathrm{~m}$ ) embedded in the track and covered with tartan. The force signals were acquired at 200 sample/s and low-pass filtered at $70 \mathrm{~Hz}$ using a 4th-order Butterworth filter. All athletes sprinted with a forefoot strike technique.

A second session involved elite athletes (group E) of the Italian National Track and Field Team, tested on their outdoor training track. Reference data were collected using a high-speed camera (Casio Exilim EX-F1, Japan; $512 \times 384$ pixels, $300 \mathrm{frame} / \mathrm{s}$ ), positioned $5 \mathrm{~m}$ away from the lane.

In both experimental sessions, each athlete was equipped with an IMU (FreeSense Sensorize, Italy) containing a 3D accelerometer and a 3D gyroscope ( $\pm 6 \mathrm{~g}$ and $\pm 500 \mathrm{deg} / \mathrm{s}$ of full range, respectively; 200 sample/s) providing linear acceleration and angular velocity components, respectively along and about the axes of a unitembedded coordinate system. The IMU data were stored in the unit. Care was paid to the fixation of the IMU on the lower back trunk (L1 level) to limit its oscillations relative to the skeleton (Forner-Cordero et al., 2008). The low lumbar area was avoided, since it is more affected by muscular masses wobbling and a memory foam material was used as an interface between the paravertebral muscles and the IMU. An ad-hoc elastic belt was used to mount the device without limiting the athletes' movements.

Force platform and IMU samples were synchronised by recording the same impulsive event (a hammer strike on one platform upon which the IMU was positioned). For group E, no interference with the athletes training schedule was permitted and, therefore, no camera-IMU synchronisation could be performed.

The experimental protocol was the same in both sessions and consisted of 20 min warm-up followed by three sprint runs of $60 \mathrm{~m}$ starting from the blocks. To avoid fatigue, a 10 min rest period was given between runs. For each athlete, two strides were analysed at $40 \mathrm{~m}$ from the start, during the maintenance phase.

Data obtained in the first session were analysed to identify features related to the foot-strike and foot-off instants of time $\left(t_{\mathrm{FS}}, t_{\mathrm{FO}}\right)$ and used to create algorithms for their automatic detection.

The acceleration and the angular velocity vectors were represented in the unit-embedded coordinate system, and their magnitude ( $a$ and $\omega$, respectively) was computed. Since increasing the number of time-series could be useful for performing features identification (Hanlon and Anderson, 2009), the first and second time derivatives of $a$ and $\omega$ were calculated. To reduce the effect of highfrequency noise on the numerical differentiation procedure, a wavelet-based approach was used to smooth the signal (Luo et al., 2006).

Each dataset of group A was time-matched with the vertical force. Thresholds of $10 \mathrm{~N}$ on the rising, and $25 \mathrm{~N}$ on the descending force signal were used to determine reference $t_{\mathrm{FS}}$ and $t_{\mathrm{FO}}$, respectively (Hunter et al., 2005). Reference stance $\left(d_{\text {stance }}=t_{\mathrm{FO}}-t_{\mathrm{FS}}\right)$ and cycle duration between subsequent $t_{\mathrm{FO}}$ of the same leg, assumed to be equal to the stride duration, $d_{\text {stride }}$, were calculated.

Repeatable quantifiable features, such as maxima, minima, or slopes, were identified in each IMU dataset of group $\mathrm{A}$, in correspondence with $t_{\mathrm{FS}}$ and $t_{\mathrm{FO}}$ Algorithms for their automatic detection were developed in Matlab (v7.9 Mathworks), and an estimate of stance ( $\left.\tilde{d}_{\text {stance }}\right)$ and stride duration $\left(\tilde{d}_{\text {stride }}\right)$ was performed.

The algorithms were tested on group $\mathrm{E}$ data using the same type of time-series dataset to estimate $\tilde{d}_{\text {stance }}$ and $\tilde{d}_{\text {stride }} . t_{\mathrm{FS}}$ and $t_{\mathrm{FO}}$ were visually identified on the videos and reference $d_{\text {stance }}$ and $d_{\text {stride }}$ calculated.

Validation of the estimated parameters was performed, for both groups, by evaluating the absolute difference between IMU estimate $\left(\tilde{d}_{\text {stance }}\right.$ and $\left.\tilde{d}_{\text {stride }}\right)$ and reference measurements $\left(d_{\text {stance }}\right.$ and $\left.d_{\text {stride }}\right): e_{\text {stance }}$ and $e_{\text {stride }}$.

The following statistical analysis was performed using SPSS (version 17.0; alpha level=0.05):

- Repeated-measures ANOVA on $\tilde{d}_{\text {stance, }}, \tilde{d}_{\text {stride }}, d_{\text {stance, }}$ and $d_{\text {stride }}$ to verify the effect of factors: group, athlete and trial;

- Descriptive statistics for $\tilde{d}_{\text {stance, }}, \tilde{d}_{\text {stride }}, d_{\text {stance, }}$ and $d_{\text {stride }}$ (mean \pm standard deviation (SD)) for independent factors;

- Test of normal distribution of $e_{\text {stance }}$ and $e_{\text {stride }}$ with the Shapiro-Wilk test $(p>0.05)$;

- Bland-Altman plots of $e_{\text {stance }}$ and $e_{\text {stride, }}$, corrected for the effect of repeated measurement error (Bland and Altman, 2007), to assess the agreement between measurement systems with multiple observations (Atkinson and Nevill, 1998);

- Inspection of these Bland-Altman plots and correlation exploration (Nevil and Atkinson, 1997) to investigate the absence of heteroscedasticity;

- Repeated-measures ANOVA on $e_{\text {stance }}$ and $e_{\text {stride }}$ to verify the effect of the factors: group, athlete and trial.

- When trial and athlete had a not significant effect on variables, absolute reliability for repeated measurement was assessed in terms of limits of agreement (LOA) (Bland and Altman, 2007).

\section{Results}

No consistent feature was detected for group A on $a$ (Fig. 1(A)), its derivatives, or $\omega$ (Fig. 1(B)) that could consistently be related to $t_{\mathrm{FS}}$ and $t_{\mathrm{FO}}$. These instants of time were instead related to positive peaks (assumed to coincide with foot-off) and negative peaks (corresponding to foot-strike) on the $\ddot{\omega}$ waveform (Fig. $1(\mathrm{C})$ ) and were used to estimate $\tilde{d}_{\text {stance }}$.

A feature-based estimate of the cycle duration was performed based on a consistent positive peak ( $\left.\omega_{\text {peak }}\right)$, detectable even by simple visual inspection in $\omega$ (Fig. 1(B)). Distance between odd $\omega_{\text {peak }}$ was used to estimate $\tilde{d}_{\text {stride }}$.

Repeated-measures ANOVA showed that all time intervals differed between groups $(p<0.05)$, but not across athletes $(p>0.05)$ and trials $(p>0.05)$ of the same group. Mean values $( \pm$ SD) for $d_{\text {stance }}$ across all athletes, trials and strides were $0.125 \mathrm{~s}( \pm 0.015)$ for group A and $0.105 \mathrm{~s}( \pm 0.010)$ for group E. Equivalent means for $d_{\text {stride }}$ were $0.495 \mathrm{~s}( \pm 0.040)$ and $0.455 \mathrm{~s}( \pm 0.015)$.

Normality and no heteroscedasticity were found for $e_{\text {stance }}$ and $e_{\text {stride }}$ (correlation $=0.01$ and 0.07 ). Bland-Altman plots indicated the agreement between reference and IMU estimates for both groups, the mean errors being close to the IMU temporal resolution (0.005 s) (Fig. 2). No statistical differences were found between groups, athletes, and trials. 95\% LOA were lower than 

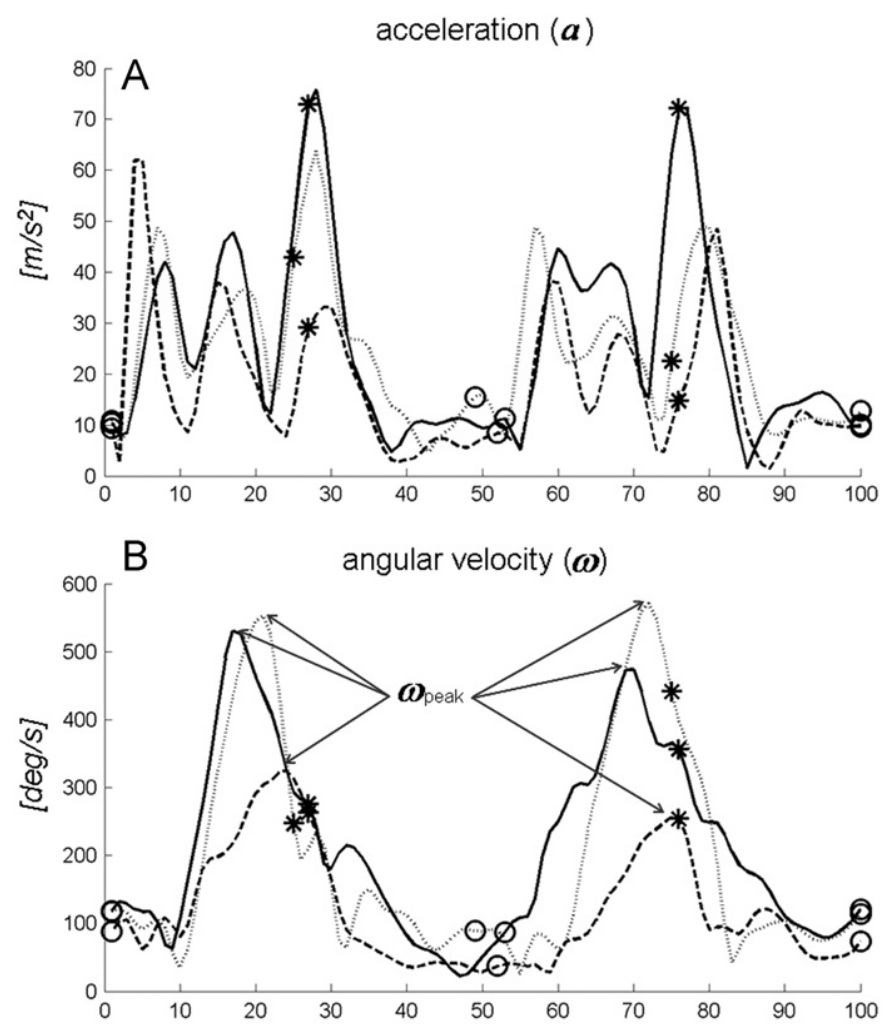

C second derivative of the angular velocity $(\ddot{\boldsymbol{\omega}})$

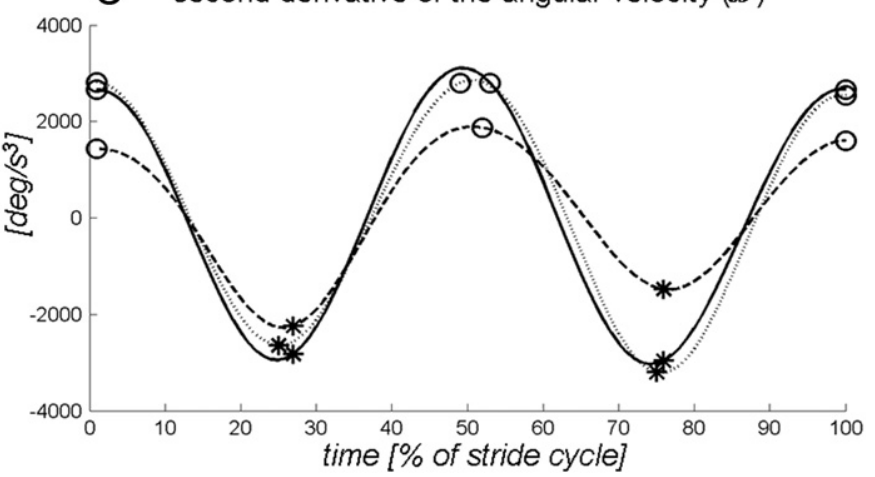

Fig. 1. (A) Acceleration $a$, (B) angular velocity $\omega$, and (C) second derivative of the angular velocity $\ddot{\omega}$ for three randomly selected athletes of group $A$, with reference to a randomly chosen stride cycle during the maintenance phase of sprint running defined as the part of the run from when the athlete reaches an upright sprinting position until the end of the run (Jones et al., 2009). Stride cycles were segmented using the force platform signals and expressed in percentage of the total cycle duration. Symbols $(O)$ and $(*)$ indicate reference foot-strike and foot-off instants of time respectively. Arrows in (C) highlight peaks in angular velocity.

$0.025 \mathrm{~s}$ for both groups. Their symmetry demonstrated the absence of systematic differences of the errors between devices.

\section{Discussion}

The feature-identification analysis performed on trunkmounted IMU signals obtained during the maintenance phase of sprint running showed that neither the magnitude of the acceleration, nor its first and second derivatives provide any consistently identifiable feature correlated with $t_{\mathrm{FS}}$ and $t_{\mathrm{FO}}$. This result is in contrast with previous studies using trunk-mounted accelerometers during distance running (Auvinet et al., 2002; Lee et al., 2010; Wixted et al., 2010). Speculation on Auvinet et al. and Wixted et al. results is limited by the absence of reference measurements to support feature detection; while the discrepancy with validated results by Lee et al. can be accounted for by both a different foot position at ground contact and the higher explosiveness of sprint running. In the referenced studies selective gait events related to heel strike could probably be detected as the ankle cannot damp/filter the shock/vibration. During a sprint, the forefoot strike entails considerable damping through the multiarticular anatomy of the foot. Moreover, the movement of the muscular masses at the trunk level is characterised by a larger translational component with respect to the rotational component which, consequently, may be assumed to affect the acceleration more than the angular velocity. It is suggested that results obtained in distance running studies using IMUs not to be automatically extended to sprint running.

The magnitude of the angular velocity signal, as well as its wavelet-mediated second derivative, are characterised by repeatable and consistent events that allow for the estimate of stride and stance duration. The angular velocity peak proved to be consistent across levels of expertise, athletes, and trials. As reported in the literature, this peak is associated to a trunk rotation about its longitudinal axis, which occurs during walking as well as during distance running (Schache et al., 2002; Saunders et al., 2005). Our results evidenced the presence of this rotation during sprint running and its potential use in estimating stride duration, but not $t_{\mathrm{FS}}$ and $t_{\mathrm{FO}}$. Such instants were consistently associated only with the positive and negative peaks of the wavelet-mediated $\ddot{\omega}$ waveform. It is worth to underline that signal inconsistencies preventing easy feature identification can be present as well in other contexts such as, for example, some types of pathological walking. Given the efficacy of this method

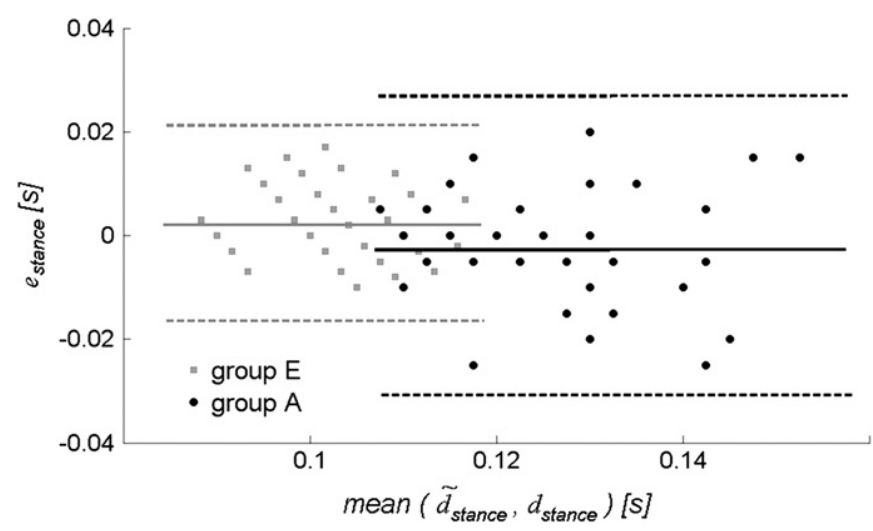

Fig. 2. Bland-Altman plots for group A and group E, representing comparisons between reference stance times and those estimated with IMU. Mean difference (solid lines) and random error lines representing 95\% limits of agreement (dashed lines) are included.

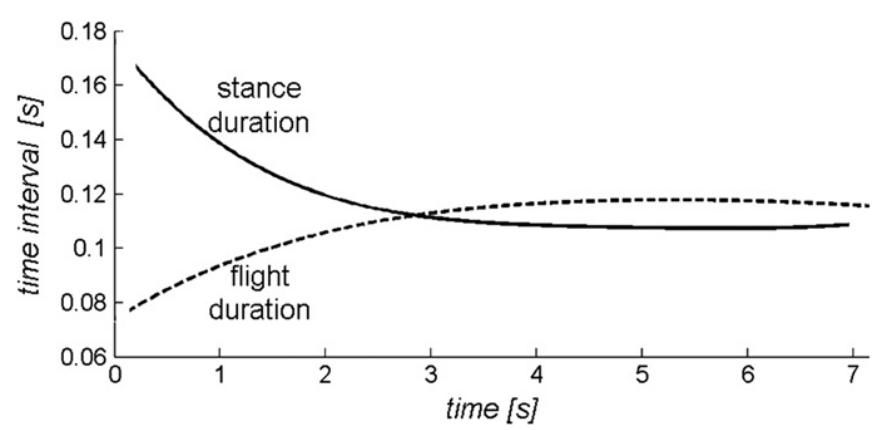

Fig. 3. Stance (solid line) and flight (dashed line) duration time-curves, as obtained from video-analysis, during a $60 \mathrm{~m}$ sprint run of a randomly chosen elite sprinter of group $\mathrm{E}$. 
on highly noisy data, there is a potential for widening its fields of application.

The absolute difference between IMU and reference measurements was of the same order as the temporal resolution of the criterion measure, as well as of the unit estimates $(0.005 \mathrm{~s})$ for both stride ( $<2 \%$ of $\tilde{d}_{\text {stride }}$ ) and stance ( $<8 \%$ of $\tilde{d}_{\text {stance }}$ ) duration. The latter result is consistent with those obtained during sprint running using shank-mounted accelerometers (Purcell et al., 2005). This error does not prevent discrimination of amateur from elite athletes' temporal parameters (the average difference of $d_{\text {stance }}$ between groups is $0.020 \mathrm{~s}$ ). It may allow the analysis of the race strategy through observation of step frequency (Salo et al., 2011) or of stance times profile (Fig. 3).

In conclusion, the proposed method presents the potential to provide reliable estimates of temporal parameters during in field training sessions. To provide coaches with reliable and automatic information, validation of the method for different phases of the sprint run during the whole race is required.

\section{Conflict of interest statement}

The authors do not have any financial or personal relationships with other people or organisations that could inappropriately influence the manuscript.

\section{Acknowledgements}

The authors wish to thank all the athletes who participated in the study and the coach Filippo di Mulo for their valuable time, Adrien Michel and Dr. Marco Donati for their practical and technical support in performing the experiments and in processing data, and John McCamley for his contribution to the refinement of the manuscript. This research was supported by a grant of the Università Italo-Francese (Call Vinci) awarded to E. Bergamini. The study sponsor had no involvement in the study design, in the collection, analysis and interpretation of data, and in the writing of the manuscript and decision to submit the manuscript for publication.

\section{References}

Atkinson, G., Nevill, A.M., 1998. Statistical methods for assessing measurement error (reliability) in variables relevant to sports medicine. Sports Medicine 26 (4), 217-238.

Auvinet, B., Gloria, E., Renault, G., Barrey, E., 2002. Runner's stride analysis: comparison of kinematic and kinetic analyses under field conditions. Science \& Sports $17,92-94$
Bland, J.M., Altman, D.G., 2007. Agreement between methods of measurement with multiple observations per individual. Journal of Biopharmaceutical Statistics 17 (4), 571-582.

Cavagna, G.A., Komarek, L., Mazzoleni, S., 1971. The mechanics of sprint running. Journal of Physiology Online 217, 709-721.

Ciacci, S., Di Michele, R., Merni, F., 2010. Kinematic analysis of the braking and propulsion phases during the support time in sprint running. Gait \& Posture 31 (2), 209-212.

Čoh, M., Tomažin, K., Štuhec, S., 2006. The biomechanical model of the sprint start and block acceleration. Physical Education \& Sport 4, 103-114.

Forner-Cordero, A., Mateu-Arce, M., Forner-Cordero, I., Alcántara, E., Moreno, J.C., Pons, J.L., 2008. Study of the motion artefacts of skin-mounted inertial sensors under different attachment conditions. Physiological Measurement 29 (4), N21-N31.

Hanlon, M., Anderson, R., 2009. Real-time gait event detection using wearable sensors. Gait \& Posture 30 (4), 523-527.

Hunter, J.P., Marshall, R.N., McNair, P.J., 2004b. Interaction of step length and step rate during sprint running. Medicine \& Science in Sports \& Exercise 36 (2), 261-271.

Hunter, J.P., Marshall, R.N., McNair, P.J., 2005. Relationships between ground reaction force impulse and kinematics of sprint-running acceleration. Journal of Applied Biomechanics 21 (1), 31-43.

Hunter, J.P., Marshall, R.N., McNair, P.J., 2004a. Reliability of biomechanical variables of sprint running. Medicine \& Science in Sports \& Exercise 36 (5), 850-861.

Ito, A., Ishikawa, M., Isolehto, J., Komi, P.V., 2006. Changes in the step width, step length, and step frequency of the world's top sprinters during a $100 \mathrm{~m}$ race. New Studies in Athletics 21 (3), 35-39.

Jones, R., Bezodis, I., Thompson, A., 2009. Coaching sprinting: expert coaches' perception of race phases and technical constructs. International Journal of Sports Science \& Coaching 4 (3), 385-396.

Kavanagh, J.J., Menz, H.B., 2008. Accelerometry: a technique for quantifying movement patterns during walking. Gait \& Posture 28 (1), 1-15.

Lafortune, M.A., Henning, E., Valiant, G.A., 1995. Tibial shock measured with bone and skin mounted transducers. Journal of Biomechanics 28 (8), 989-993.

Lee, J.B., Mellifont, R.B., Burkett, B.J., 2010. The use of a single inertial sensor to identify stride, step, and stance durations of running gait. Journal of Science and Medicine in Sport 13 (2), 270-273.

Luo, J., Bai, J., Shao, J., 2006. Application of the wavelet transforms on axial strain calculation in ultrasound elastography. Progress in Natural Science 16 (9), 942-947.

Nevill, A.M., Atkinson, G., 1997. Assessing agreement between measurements recorded on a ratio scale in sports medicine and sports science. British Journal of Sports Medicine 31 (4), 314-318.

Purcell B., Channels J., James D., Barrett R. Use of accelerometers for detecting footground contact time during running. Proceedings of SPIE 2005; 6036(603615): $1-8$.

Salo, A.I., Bezodis, I.N., Batterham, A.M., Kerwin, D.G., 2011. Elite sprinting: are athletes individually step-frequency or step-length reliant? Medicine \& Science in Sports \& Exercise 43 (6). 1055-1062.

Saunders, S.W., Schache, A., Rath, D., Hodges, P.W., 2005. Changes in three dimensional lumbo-pelvic kinematics and trunk muscle activity with speed and mode of locomotion. Clinical Biomechanics (Bristol, Avon) 20 (8), 784-793.

Schache, A.G., Blanch, P., Rath, D., Wrigley, T., Bennell, K., 2002. Three-dimensional angular kinematics of the lumbar spine and pelvis during running. Human Movement Science 21 (2), 273-293.

Verkerke, G.J., Hof, A.L., Zijlstra, W., Ament, W., Rakhorst, G., 2005. Determining the centre of pressure during walking and running using an instrumented treadmill. Journal of Biomechanics 38 (9), 1881-1885.

Wixted, A.J., Billing, D.C., James, D.A., 2010. Validation of trunk mounted inertial sensors for analysing running biomechanics under field conditions, using synchronously collected foot contact data. Sports Engineering 12, 207-212. 